\title{
Morphological and Morphometric Analysis of Vertebra Prominens with Reference to Spinous Process and its Clinical Implications
}

\author{
Padma Priyaa. $\mathrm{N}^{1}$ and Karthik Ganesh Mohanraj ${ }^{2}$ \\ ${ }^{1}$ Saveetha Dental College and Hospitals,, Saveetha Institute of Medical and Technical \\ Sciences (SIMATS), Saveetha University, Chennai - 600077, India \\ ${ }^{2}$ Assistant Professor Department of Anatomy, Saveetha Dental College and Hospitals,, Saveetha Institute of \\ Medical and Technical Sciences (SIMATS), Saveetha University, Chennai - 600077, India
}

\section{ABSTRACT}

Anatomy of the pedicles of the seventh cervical vertebra (C7) at the cervicothoracic junction isn't like other cervical vertebrae. Fixation of $\mathrm{C7}$ is required at some point of cervical vertebra and top thoracic injuries in clinical practice.The 7th cervical (neck) vertebra (C7) is also the outstanding vertebra due to the length of its spinous manner (the projection off the again of the vertebral body).The spinous method of the top thoracic vertebra (T1) just below C7 is every now and then even greater outstanding than that of the vertebra prominens. However, the everyday pedicle screw insertion methods may additionally have troubles in clinical practice primarily based on the anatomical features of C7. In everyday spinal surgical operation practice, correct interpretation of the information provided from computed tomography (CT) imaging is very critical to visualize the applicable bone anatomy and make accurate decisions. This observe is to explore a brand new pedicle screw insertion method for C7 and to provide anatomical and radiographic basis for clinical application. From the present study the total length of the vertebra is calculated, the length from the vertebral foramen to the spinous process is calculated ,the breadth of the middle part of the spinous process is calculated, and the breadth of the tip of the spinous process is calculated and their mean values and their standard deviation is calculated accordingly.

KEY WORDS: MORPHOMETRY, CERVICAL VERTEBRA, VERTEBRA PROMINENS, SPINOUS PROCESS, CLINICAL SIGNIFICANCE.

\section{INTRODUCTION}

Vertebra prominens is also known as the seventh cervical vertebra. One of the uniqueness of this vertebra is that it has a long and prominent spinous process which can be felt from the skin surface, hence the name is vertebra

\section{ARTICLE INFORMATION}

*Corresponding Author: karthikm.sdc@saveetha.com Received 1st August 2020 Accepted after revision 30th Sep 2020 Print ISSN: 0974-6455 Online ISSN: 2321-4007 CODEN: BBRCBA

Thomson Reuters ISI Web of Science Clarivate Analytics USA and Crossref Indexed Journal

\section{Clarivate
Analytics}

NAAS Journal Score 2020 (4.31) SJIF: 2020 (7.728)

A Society of Science and Nature Publication,

Bhopal India 2020. All rights reserved.

Online Contents Available at: http//www.bbrc.in/

Doi: http://dx.doi.org/10.21786/bbrc/13.8/115 prominence. The seventh cervical vertebra (C7) is also the longest cervical vertebra due to the length of its spinous manner which is one of the atypical features of this vertebra. The spinous method of the top thoracic vertebra (T1) just below C7 is every now and then even greater outstanding than that of the vertebra prominens (Schilling, 2011). The spinous process is brief and bifid, the 2 divisions being regularly of unequal size (Pranati, Babu and Ganesh, 2017). Because the spinous methods are so short, positive superficial muscles (the trapezius and splenius capitis) attach to the nuchal ligament rather than directly to the vertebrae; the nuchal ligament itself attaching to the spinous approaches of $\mathrm{C} 2-\mathrm{C} 7$ and to the posterior tubercle of the atlas (Gray, 2018). 
C7 possesses the standard cervical vertebral capabilities however has some distinct features are spinous technique leads to a rounded tubercle and isn't bifid, C7 transverse foramina are small, and do now not transmit the vertebral artery, (Seppan et al., 2018) C7 anterior tubercle is small, and is the site of attachment for scalenus pleuralis and supra pleural membrane, C8 nerve, which does not have an related cervical vertebra, exits in the C7-T1 vertebral foramen under C7 (Stamatakis, 2000), C7 may also own a cervical rib, $\mathrm{C} 7$ foramen can also transmit the posterior vertebral vein (if doubled), and might transmit the gray ramus from the inferior cervical ganglion to the anterior ramus of C7, vertebral artery may additionally input at this level (typically it enters the cervical transverse foramen at C6) (Matshes and Juurlink, 2004).

Vertebra prominens (C7) is characterized as the most prominent among the cervical vertebrae, which makes it an anatomical landmark for the recognition of other spinous processes (Stonelake, Burwell and Webb, 1988). Unlike the notched spinous techniques of the C3-C6 vertebrae, the vertebra prominens resemble the $\mathrm{T} 1$ vertebra with a spinous method that is large, straight, and flattened on the give up. The give up of the nuchal ligament, which supports the muscle tissues of the neck and connects the occipital bone of the cranium to the C7 vertebra, attaches at the tip of the spinous system. The spinous method of $\mathrm{C} 7$ generally projects directly posteriorly. Unlike normal cervical vertebrae, the spinous method of C7 isn't always bifid (Ranasinghe, Yuvaraj Babu and Mohanraj, 2018). The funicular part of the ligamentum nuchae attaches to the unmarried posterior tip of the C7 spinous system (Xu et al., 1995). The muscular tissues connected to the spinous method are Trapezius, Rhomboid minor, Serratus posterior superior, Splenius capitis, Spinalis cervicis, Semispinalis thoracis, Multifidus Thoracis, Interspinales. The aim of the present study was to analyze the morphology and the morphometrical analysis of vertebra prominence and to understand its clinical importance.

\section{MATERIAL AND METHODS}

A total of 33 dried human cervical vertebra (C7) were collected from the Department of Anatomy of Saveetha Dental College and from the Institute of Anatomy, Madras Medical College, Chennai. All C7 vertebrae are numbered serially and arranged. Any broken and abnormal bones were rejected from study. The morphology and morphometry of $\mathrm{C} 7$ vertebrae are analyzed with reference to its spinous process. From the present study the total length of the vertebra prominens (TL), the length from the vertebral foramen to the spinous process (VL), the breadth of the middle part of the spinous process (VB) and the breadth of the tip of the spinous process (SB) were measured using a sliding vernier caliper. The photograph of the C7 vertebrae depicting its morphological aspects are shown in Figure 1. All the readings were noted and transferred to the Excel sheet. The mean values and their standard deviation are calculated accordingly. The obtained mean values are represented graphically with \pm Standard deviation (SD).

\section{RESULTS AND DISCUSSION}

The analysed data showed that the mean total length of the vertebra prominens (TL) was found to be $36.92 \pm$ $0.075 \mathrm{~mm}$. The mean length from the vertebral foramen to the spinous process (VL) was $10.48 \pm 0.066 \mathrm{~mm}$. The mean breadth of the middle part of the spinous process (VB) was found to be $7.61 \pm 0.034 \mathrm{~mm}$. The mean breadth of the tip of the spinous process (SB) was $8.14 \pm 0.037$ $\mathrm{mm}$. The mean of all the morphometric measurements of C7 vertebrae are shown in Figure 2.

Figure 1: Photograph of superior view of vertebra prominens showing the various dimensions measured. The total length of the vertebra prominens (TL) indicated by white arrow line, the length from the vertebral foramen to spinous process (VL) indicated by yellow arrow line, the breadth of the middle part of the spinous process (VB) indicated by red arrow line and the breadth of the tip of the spinous process (SB) indicated by black arrow line are shown here.

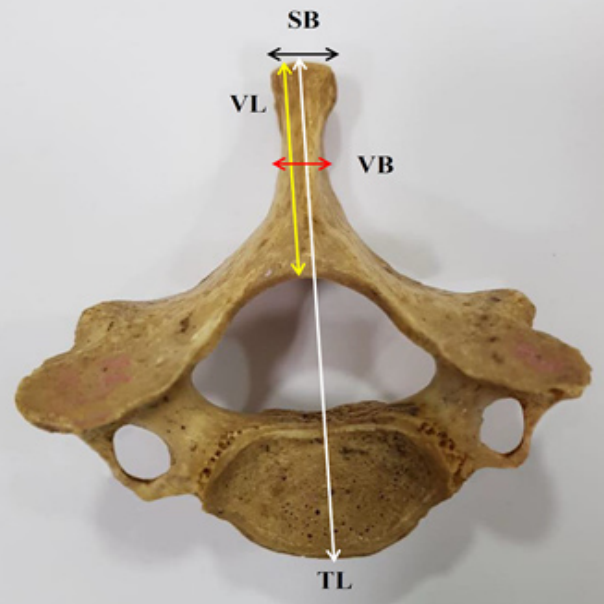

Figure 2: Graph showing the mean of all the morphometric measurements of $\mathrm{C} 7$ vertebrae. The total length of the vertebra prominens $(\mathrm{TL})$, the length from the vertebral foramen to the spinous process (VL), the breadth of the middle part of the spinous process (VB) and the breadth of the tip of the spinous process (SB) are expressed as Mean \pm SD in $\mathrm{mm}$.

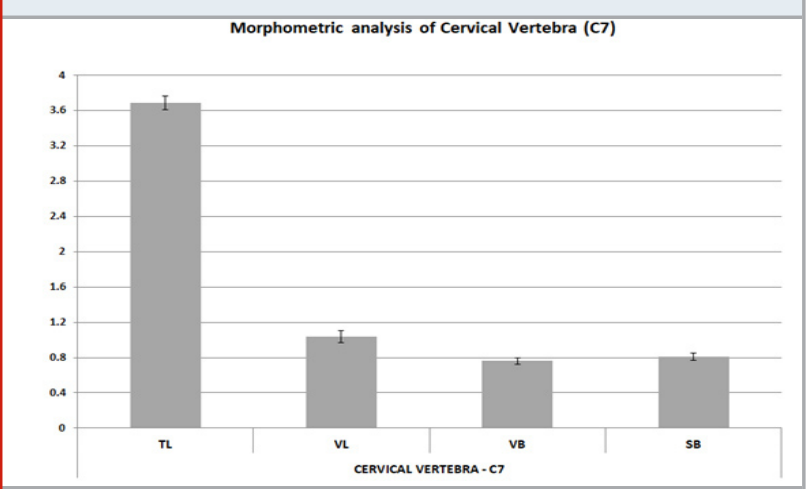


The vertebra prominens, or $\mathrm{C} 7$, has an exceptionally long and outstanding spinous procedure, that's palpable from the skin surface. As a variation, seventh cervical vertebra is associated with an abnormal more rib, known as a cervical rib, which develops from the anterior root of the transverse process (Muthukumar and Mohanraj, 2019). These ribs are typically small, but may now and again compress blood vessels (such as the subclavian artery or subclavian vein) or nerves within the brachial plexus, causing pain, numbness, tingling, and weakness in the top limb, a condition known as thoracic outlet syndrome (Ravichandran, Yuvraj Babu and Mohanraj, 2018). Very rarely, this rib takes place in a pair (Umakanth, Babu and Mohanraj, 2018).

The lengthy spinous manner of C7 is thick and nearly horizontal in direction. It is not bifurcated, and leads to a tubercle that the ligamentum nuchae attaches (Mary, Babu and Mohanraj, 2018). This manner isn't usually the most distinguished of the spinous processes, being found most effective about 70\% of the time, C6 or T1 can every now and then be the most outstanding

(Greiner, 2017).

While C7 vertebra is anatomically very just like thoracic vertebra, the cervicothoracic junction presents several demanding situations to the backbone general practitioner in operation settings(Shahzan, Babu and Mohanraj, 2018). The transition from the mobile, lordotic cervical backbone to the fixed, kyphotic, thoracic segment, (Keskin et al., 2015)Relative lack of epidural area and the improved medial angulation wanted for pedicle cannulation at C7 and essential adjoining neural and vascular structures at these degrees make surgical intervention more challenging and probably hazardous(Jang et al., 2011).

In everyday spinal surgical operation practice, ok interpretation of the information provided from computed tomography (CT) imaging is very critical to visualize the applicable bone anatomy and make accurate decisions(Kumar, Babu and Mohanraj, 2018). This situation is specially valid in the C7 vertebra, wherein morphometric measurements are essential elements that may restrict surgical success(Vignesh, Babu and Mohanraj, 2018). Skeletally mature teenagers and its C7 vertebra should injure mainly in serious unstable traumas, neoplastic or infectious lesions (Ramieri et al., 2011).

\section{CONCLUSION}

From the present study the morphometric parameters and the variation of vertebra prominens are analysed. The clear understanding and knowledge on its morphometry and occasional variations may be helpful for surgeons and other clinicians dealing with this region. It also provides valuable information and orientation for orthopedicians involved in the surgical and other treatment procedures. Thus within the limitations of the study, we can conclude that the morphological and morphometrical analysis of vertebra prominens has several clinical implications which were emphasised from the present study.

\section{ACKNOWLEDGEMENTS}

Nil

Conflict of Interest: None declared.

\section{REFERENCES}

Gray, H. (2018) Anatomy, Descriptive and Surgical. Creative Media Partners, LLC.

Greiner, T. M. (2017) 'Shape analysis of the cervical spinous process', Clinical anatomy, 30(7), pp. 894900.

Jang, W. Y. et al. (2011) 'A computed tomography-based anatomic comparison of three different types of $\mathrm{c7}$ posterior fixation techniques : pedicle, intralaminar, and lateral mass screws', Journal of Korean Neurosurgical Society, 50(3), pp. 166-172.

Keskin, F. et al. (2015) 'Radiological anatomy of the C7 vertebra: Clinical implications in spine surgery', Journal of craniovertebral junction and spine, 6(1), pp. 30-35. Kumar, M., Babu, K. Y. and Mohanraj, K. G. (2018) 'Hair fall due to stress factors-A survey', Drug Invention Today, 10. Available at: https://bit.ly/33Nba1T

Mary, D., Babu, K. Y. and Mohanraj, K. G. (2018) 'Craniometric analysis of foramen magnum with reference to occipital condyles for the determination of sex using dry human skulls', Drug Invention Today, 10(10). Available at: https://bit.ly/2HnE7KB.

Matshes, E. W. and Juurlink, B. (2004) Human Osteology and Skeletal Radiology: An Atlas and Guide. CRC Press.

Muthukumar, A. and Mohanraj, K. G. (2019) 'Prevalence, knowledge, and awareness of thyroid disorders among young adult women population', Drug Invention Today, 11(10). Available at: https://bit.ly/2ZVelU5

Pranati, T., Babu, K. Y. and Ganesh, K. (2017) 'Assessment of plantar arch index and prevalence of flat feet among south Indian adolescent population', Research journal of pharmaceutical, biological and chemical sciences. Journal of Pharmaceutical Sciences and Research, 9(4), p. 490.

Ramieri, A. et al. (2011) 'Spine surgery in neurological lesions of the cervicothoracic junction: multicentric experience on 33 consecutive cases', European Spine Journal, pp. 13-19. doi: 10.1007/s00586-011-1748-z.

Ranasinghe, A. N., Yuvaraj Babu, K. and Mohanraj, K. G. (2018) 'Estimation of orbital index for gender determination', Drug Invention Today, 10(12). Available at: https://bit.ly/3iT6JsP

Ravichandran, S., Yuvraj Babu, K. and Mohanraj, K. G. (2018) 'Correlation of facial and nasal index in gender determination', Drug Invention Today, 10(12). Available 
at: https://bit.ly/3hNgBTK

Schilling, N. (2011) 'Evolution of the axial system in craniates: morphology and function of the perivertebral musculature', Frontiers in zoology, 8(1), p. 4.

Seppan, P. et al. (2018) 'Therapeutic potential of Mucuna pruriens (Linn.) on ageing induced damage in dorsal nerve of the penis and its implication on erectile function: an experimental study using albino rats', The Aging Male, pp. 1-14. doi: 10.1080/13685538.2018.1439005.

Shahzan, M. S., Babu, K. Y. and Mohanraj, K. G. (2018) 'Estimation of stature using clavicle bone', Drug Invention Today, 10. Available at: https://bit. ly/2ZRE6oo

Stamatakis, J. D. (2000) 'Applied Radiological Anatomy P. Butler, A. W. M. Mitchell and H. Ellis (eds) $282 \times 224$ mm. Pp. 441. Illustrated. 1999. Cambridge: Cambridge University Press', British Journal of Surgery, pp.
381-381. doi: 10.1046/j.1365-2168.2000.01370-2.x. Stonelake, P. S., Burwell, R. G. and Webb, J. K. (1988) 'Variation in vertebral levels of the vertebra prominens and sacral dimples in subjects with scoliosis', Journal of anatomy, 159, pp. 165-172.

Umakanth, K., Babu, K. Y. and Mohanraj, K. G. (2018) 'Morphological and morphometrical analysis of Chassaignac's tubercle in dry human cervical vertebrae', Drug Invention Today, 10(10). Available at: https://bit. ly/2FXj7t6

Vignesh, P., Babu, K. Y. and Mohanraj, K. G. (2018) 'Morphometric analysis of gonial angle and mandibular ramus measurement as predictors of sex and age in dry human mandibles', Drug Invention Today, 10(10). Available at: https://bit.ly/3iOl7CM

$\mathrm{Xu}, \mathrm{R}$. et al. (1995) 'Morphology of the second cervical vertebra and the posterior projection of the C2 pedicle axis', Spine, 20(3), pp. 259-263. 